\title{
LYCEN 2001-20
}

\section{Rescattering corrections in elastic scattering}

December 17, 2018

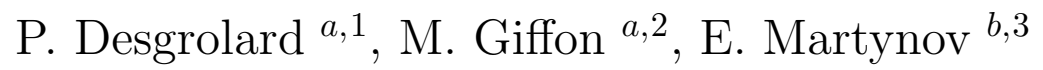

${ }^{a}$ Institut de Physique Nucléaire de Lyon, IN2P3-CNRS et Université C. Bernard, 43 boulevard du 11 novembre 1918, F-69622 Villeurbanne Cedex, France

${ }^{b}$ Bogolyubov Institute for Theoretical Physics, National Academy of Sciences of Ukraine, 03143 Kiev-143, Metrologicheskaja 14b, Ukraine

\begin{abstract}
A detailed study of the rescattering series is performed within a model, using a generalized procedure of eikonalization and fitted to the $p p$ and $\bar{p} p$ elastic scattering data. We estimate and compare the various rescattering corrections to be added to the Born contribution in the amplitude. We find that their number is finite, whereas it increases with the energy and the transfer, like does their importance. In the domain where data exist, we find also that a correct computation must include, at least, all two- and three-Reggeon exchanges and some four- and five-Reggeon exchanges. Any approximation aiming to reduce this (large) number of exchange would be hazardous, especially when extrapolating. We extend our estimates in the domain of future experiments.
\end{abstract}

\footnotetext{
${ }^{1}$ E-mail: desgrolard@ipnl.in2p3.fr

${ }^{2}$ E-mail: giffon@ipnl.in2p3.fr

${ }^{3}$ E-mail: martynov@bitp.kiev.ua
} 


\section{Introduction}

The different aspects of Reggeon rescattering (or Regge cuts) have been investigated since the pioneering work of Gribov [1], who first developed a Regge calculus. A well defined procedure for determining individual diagrams corresponding to any multi-Reggeon exchanges has been developed in [2]. However these works and the following ones have been devoted mainly either to the study of analytical forms for the cuts or to the study of various summation schemes of multi-Reggeon diagrams [3]. Even when all the rescattering corrections were taken into account in the fits 4 , 5 to experimental data, as a rule, determining the relative importance of various individual $n$-Reggeon exchange contributions has not received much interest (see however in [6] where this aspect is discussed).

Motivated by the experiments prospected [7] at RHIC and LHC, intended to measure the conventional observables in new ranges of energy and transfer, we are concerned by the following question. How should we take into account the rescattering corrections to the Born approximation in a correct computation of those observables ?

To answer this question, one generally uses an eikonalization procedure which is also a remedy to cure the shortcoming of amplitudes violating the Froissart-Martin bound [8] at the Born level. However such a procedure is not unique and generally it involves a numerical integration in the Fourier-Bessel's transform of the eikonalized amplitude over the impact-parameter (" $b ")$. Furthermore the eikonalization, as a global process, hides the physical origin in terms of "Reggeon" exchanges t.

Our aim is to investigate numerically the rescattering corrections to the Born approximation : their relative importance, their physical meaning in terms of various Reggeon exchanges and their minimal number required by a correct reproduction of experimental data. For that purpose, we use a so-called generalized eikonalization (GE) procedure [9] recently applied to Regge models (e.g. [5])and fitted to elastic $p p$ and $\bar{p} p$ scattering data.

In the amplitude describing the scattering process in the squared energy-transfer $(s, t)$ space, we can separate the Born contribution and the rescattering series

$$
\begin{aligned}
A_{p p}^{\bar{p} p}(s, t) & =A_{p p ; G E}^{\bar{p} p}(s, t) \\
& =a_{p p ; B o r n}^{p p}(s, t)+A_{p p, \text { rescat }}^{\bar{p} p}(s, t)
\end{aligned}
$$

with

$$
A_{p p, r e s c a t}^{\bar{p} p}(s, t)=\sum_{n_{+}=0}^{\infty} \sum_{n_{-}=0}^{\infty} a_{p p ; n_{+}, n_{-}}^{\bar{p} p}(s, t) .
$$

Each term of this series (2) is analytically known for the models under interest. An example of driving the calculations is indicated in the Appendix fo We shall demonstrate that the series is conveniently approximated with a finite (although not small) number of terms. This possible truncation allows an easy study of the

\footnotetext{
${ }^{4}$ We affect the generic name "Reggeon" to any component of the elastic scattering amplitude we discuss for $p p$ and $\bar{p} p$ process i.e. Pomeron and Odderon as well as $f$ - and $\omega$-subleading trajectories.

${ }^{5}$ The calculation of the non-truncated series is performed as in [5] within the GE procedure [9].
} 
number and specificity of the exchanges that we must keep in the infinite summation to obtain a good accuracy in the final evaluation of the observables : the total crosssection, $\sigma_{\text {tot }}$, the ratio of the forward real to imaginary parts of the amplitude, $\rho$, the differential cross section, $\frac{d \sigma}{d t}$. Furthermore, with a suitably chosen Born amplitude, it avoids the time consuming numerical integration, generally required by any complete eikonalization procedure.

Each component of the series is labelled with two indexes $\left(n_{ \pm}\right)$, each of them having a specific physical meaning. It is straightforward to constat that the first contribution to the rescattering series $(2)$, with $\left(n_{+}, n_{-}\right)=(0,0)$, is a sum of all diagrams of two-"Reggeon" exchanges. In fact, this $(0,0)$ term involves ten exchanges so different as Pomeron-Pomeron, Pomeron- $f$, Pomeron-Odderon, Pomeron- $\omega, f$ - $f$, $f$-Odderon, $f-\omega$, Odderon-Odderon, Odderon- $\omega, \omega-\omega$. It is easy to see that any term, with $n_{+}+n_{-}=N$, is the sum of all diagrams with $N+2$ Reggeons. We have no theoretical argument to sort out the magnitude of terms entering in (2), even when $N$ is as small as 1 . When $N \gg 1$ many terms are included in the summation, with alternated signs inducing many cancellations. So, a careful numerical examination is interesting to find those exchanges which are the most important.

For the present estimation, we adopt the final amplitude of [5], which corresponds to a so-called "Dipole Pomeron" (DP) model [ with the GE method.

Such a choice has been made because it is a recently published amplitude respecting the Froissart-Martin bound, implying an involved formalism for the most general treatment up to now (to our knowledge) of the eikonalization process including 3 added free parameters. This complication may of course obscure the physical sense, but the fit is satisfying for the forward and non-forward data up to the largest explored $|t|$ (14 $\mathrm{GeV}^{2}$ at the ISR, neighboring the Regge limit of application). It is, on our opinion, necessary to account data in the widest range of high energies and transfers to get confidence on predictive power outside the fitted sets of data (remember, the TOTEM project [7] plans measurements at the Large Hadron Collider up to at least $|t|=10 \mathrm{GeV}^{2}$ and it is precisely the LHC -or Tevatron or RHICenergy the most interesting to discuss at present).

The results we found (driven entirely by an analytical calculation) have not only an illustrative character, we have checked that our conclusions would be also valid for a "Monopole Pomeron" version, as used in [11], with this GE procedure.

\section{Rescatterings and amplitude}

To estimate the rescattering effects, in an absolute manner, we choose to plot the quantities (appearing basically as the most convenient)

$$
\Re \text { e and } \Im \mathrm{m}\left[S_{n_{+}, n_{-}}\right], \quad S_{n_{+}, n_{-}}=a_{p p ; B o r n}(s, t)+a_{p p ; n_{+}, n_{-}}(s, t),
$$

${ }^{6}$ Actually in this model the Pomeron "dipole", linear combination of a simple and a double pole in the angular momentum $J$-plane, as explicated in [10], is complemented by 2 standard Reggeons, $f$ and $\omega$, by an Odderon dipole conveniently multiplied by an exponential damping factor. 
for $t=0$ and for some representative $t$ 's. We can easily compare the rescattering corrections with the results of the computations at the Born level and with the complete GE. We obtain, for the DP amplitude borrowed in [5], Figs. 1-6 yielding, on a linear scale 凹, the imaginary (left) and real (right) parts of (3), labelled by $+\left(n_{+}, n_{-}\right)$and plotted versus the energy $\sqrt{s}$, (including the highest projected LHC energy) for six selected values of $t=0 .,-0.5,-1 .,-2 .,-5 .,-10$. $\mathrm{GeV}^{2}$.

Alternatively, to estimate the rescattering effects relatively to the Born result, we may also rewrite the imaginary part of the amplitude
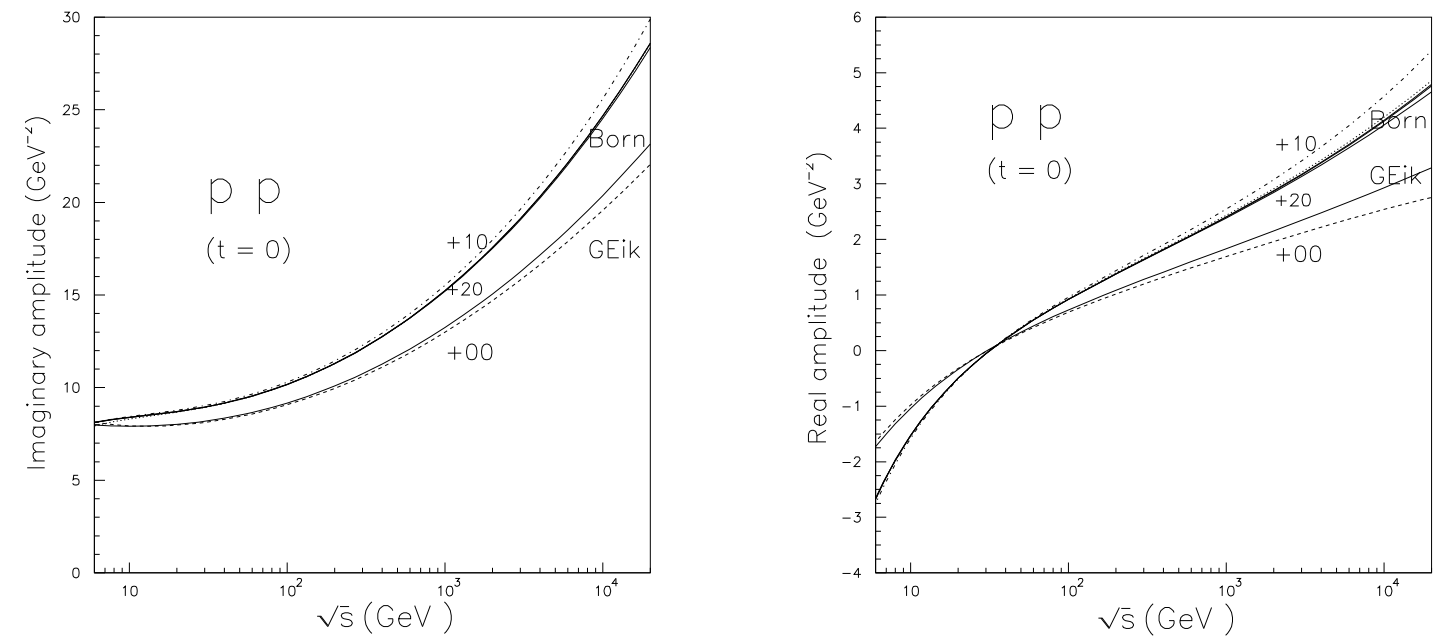

Figure 1: Separate contributions of the main rescattering corrections (see the text) added to the Born result in the imaginpackfig/ary (left) and real (right) part of the $t=0$ amplitude $\left(S_{n_{+}, n_{-}}(3)\right.$, in dashed lines, labelled by $\left.+\left(n_{+}, n_{-}\right)\right)$. Also shown in solid lines are the pure Born amplitude (Born) and the eikonalized amplitude, once the complete generalized eikonalization is performed (GEik).

$$
\Im \mathrm{m}\left(A_{p p}^{\bar{p} p}(s, t)\right)=\Im \mathrm{m}\left(a_{p p ; B o r n}^{\bar{p} p}(s, t)\right)\left(1+\sum_{n_{+}=0}^{\infty} \sum_{n_{-}=0}^{\infty} R_{n_{+}, n_{-}}^{(\mathrm{im})}\right),
$$

and use this form to settle a hierarchy among the different terms. Explicitly,

$$
R_{n_{+}, n_{-}}^{(\mathrm{im})}=\frac{\Im \mathrm{m}\left(a_{p p ; n_{+}, n_{-}}(s, t)\right)}{\Im \mathrm{m}\left(a_{p p, \text { Born }}^{\bar{p} p}(s, t)\right)}
$$

and similarly for the real part of the relative rescattering term, defining $R_{n_{+}, n_{-}}^{(\mathrm{re})}$.

We list in Table 1 an example of these estimations, for the first values of the indexes $\left(n_{+}, n_{-}\right)$(we limit somewhat arbitrarily $R^{(\mathrm{im})}$ and $R^{(\mathrm{re})}$ to one percent). The examination of the results, shown in Figs. 1-6 and Table 1, calls for the following comments with increasing $|t|$ :

\footnotetext{
7 For commodity in the visualization on a linear scale, the amplitudes with their original normalization [5] have been divided here by $s$.
} 

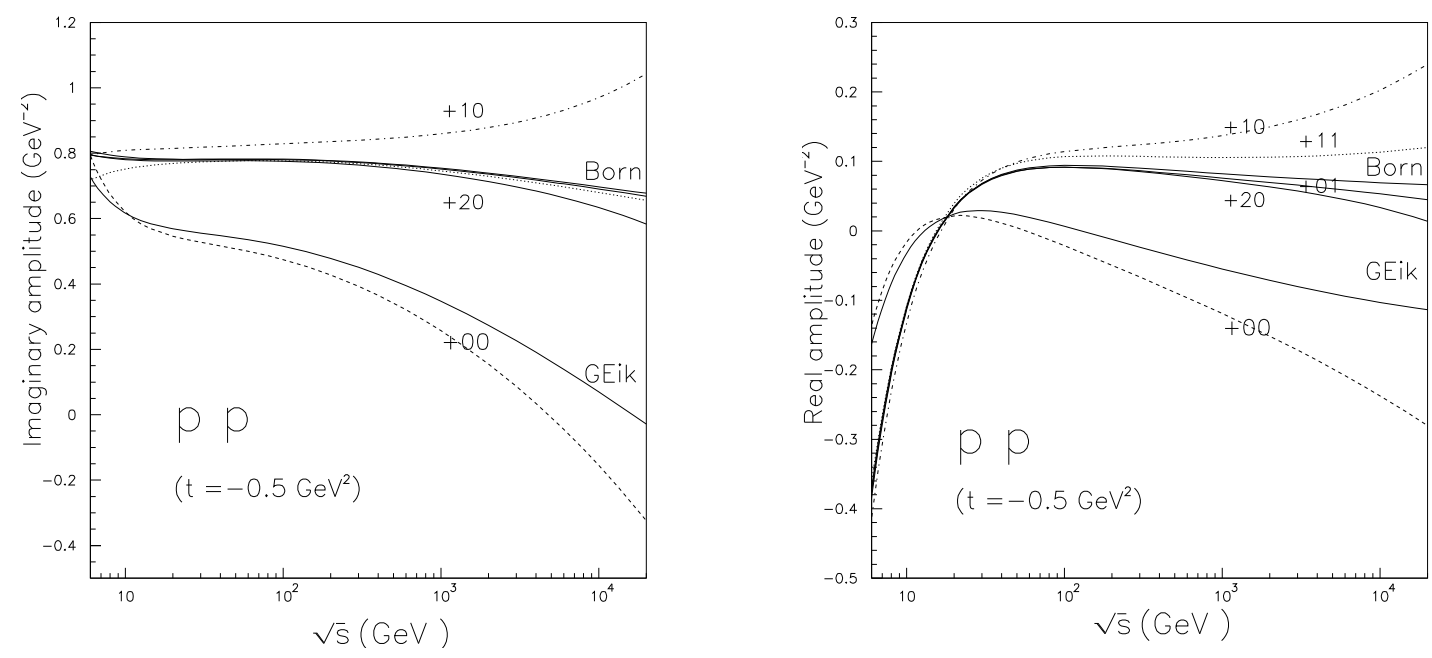

Figure 2: Same as in Fig.1 for $t=-0.5 \mathrm{GeV}^{2}$
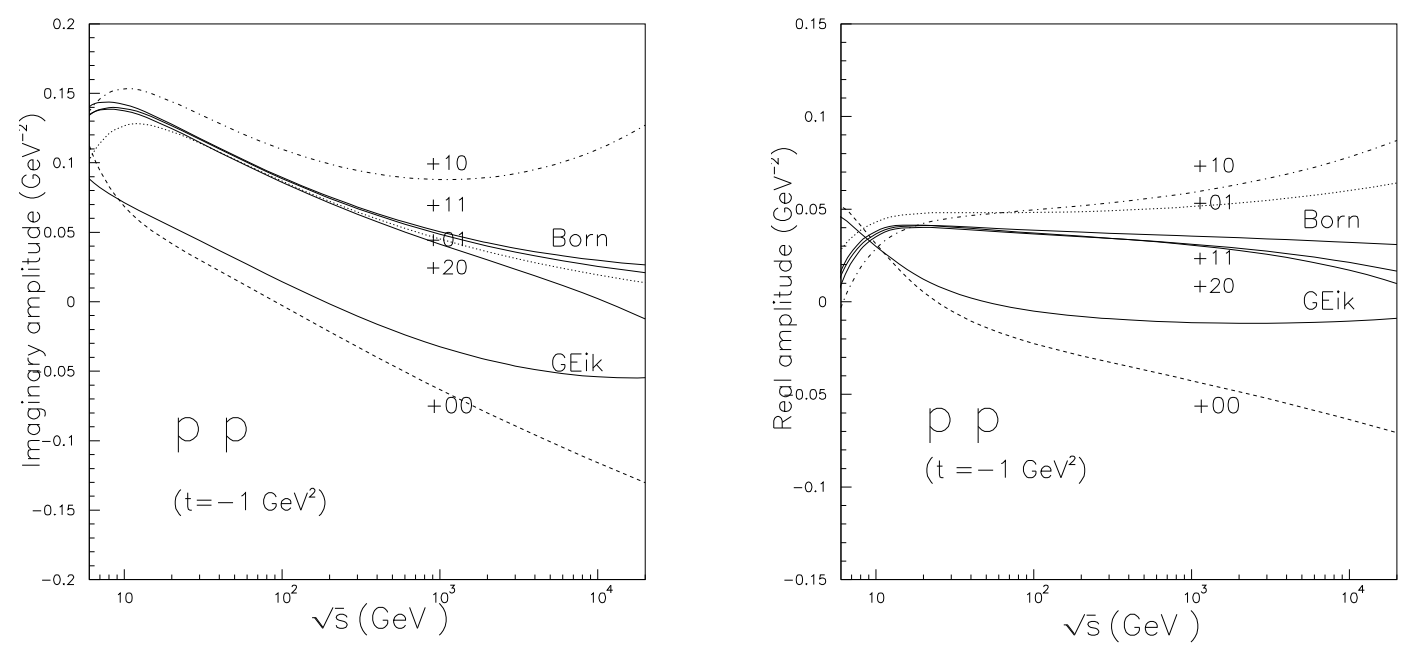

Figure 3: Same as in Fig.1 for $t=-1 . \mathrm{GeV}^{2}$

(1) when $t=0$ (see Fig, 1), adding separate corrections for $n_{+}>2, n_{-}>0$ to the Born term gives curves that cannot be distinguished by eye from the Born one. In other terms, a good approximation of the rescattering series is achieved by keeping only the terms $\left(n_{+}, n_{-}\right)=(0,0),(1,0),(2,0)$. We can see that the rescattering corrections increase with the energy and we remark the change of sign and of scale shown by the real part, as required to account for the experimental characteristics of the $\rho$-ratio.

(2) when $t$ is in the first diffraction cone (typically $t=-0.5 \mathrm{GeV}^{2}$, see Fig. 2), in addition to the three terms already listed for the forward amplitude, the terms with $\left(n_{+}, n_{-}\right)=(1,1),(0,1)$ bring small contributions to the amplitude which increase with the energy like the three other ones. Like in the forward case, the real and imaginary parts of the eikonalized amplitude are below the corresponding Born ones.

(3) when $t=-1 \mathrm{GeV}^{2}$ (see Fig. 3) in the vicinity of the dip seen in the $p p$ 

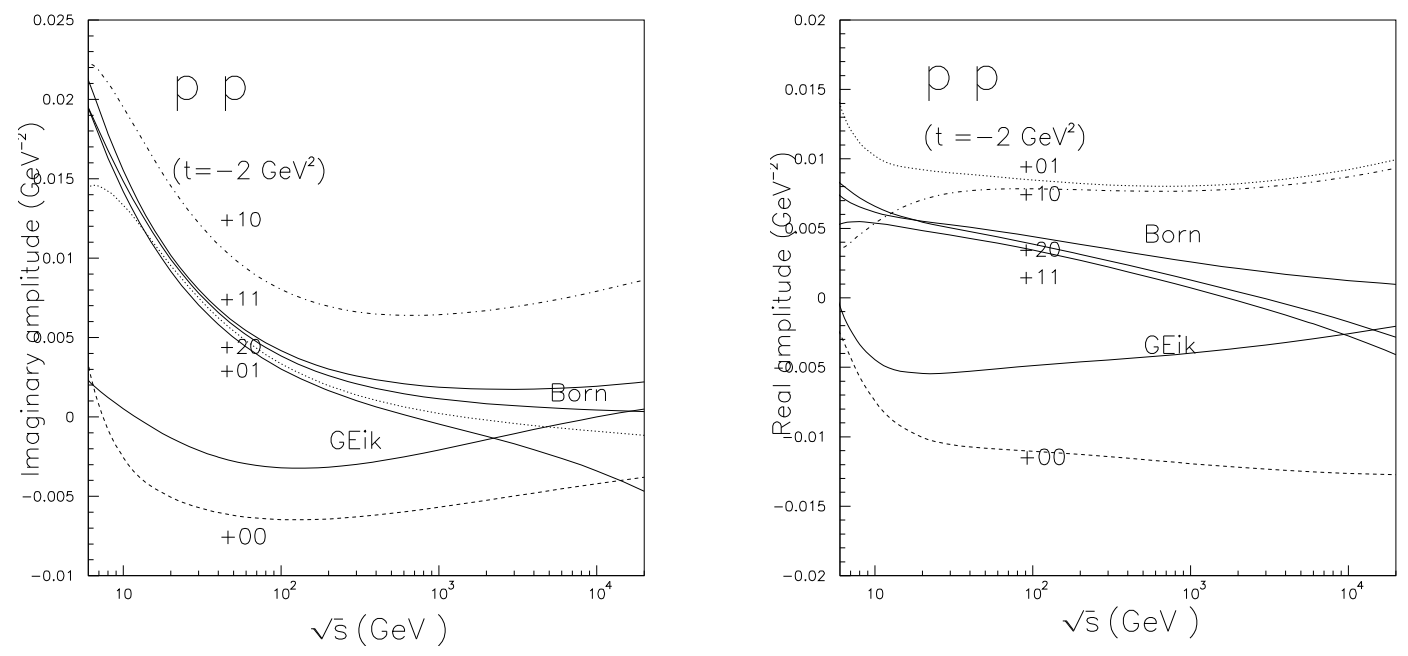

Figure 4: Same as in Fig.1 for $t=-2 . \mathrm{GeV}^{2}$
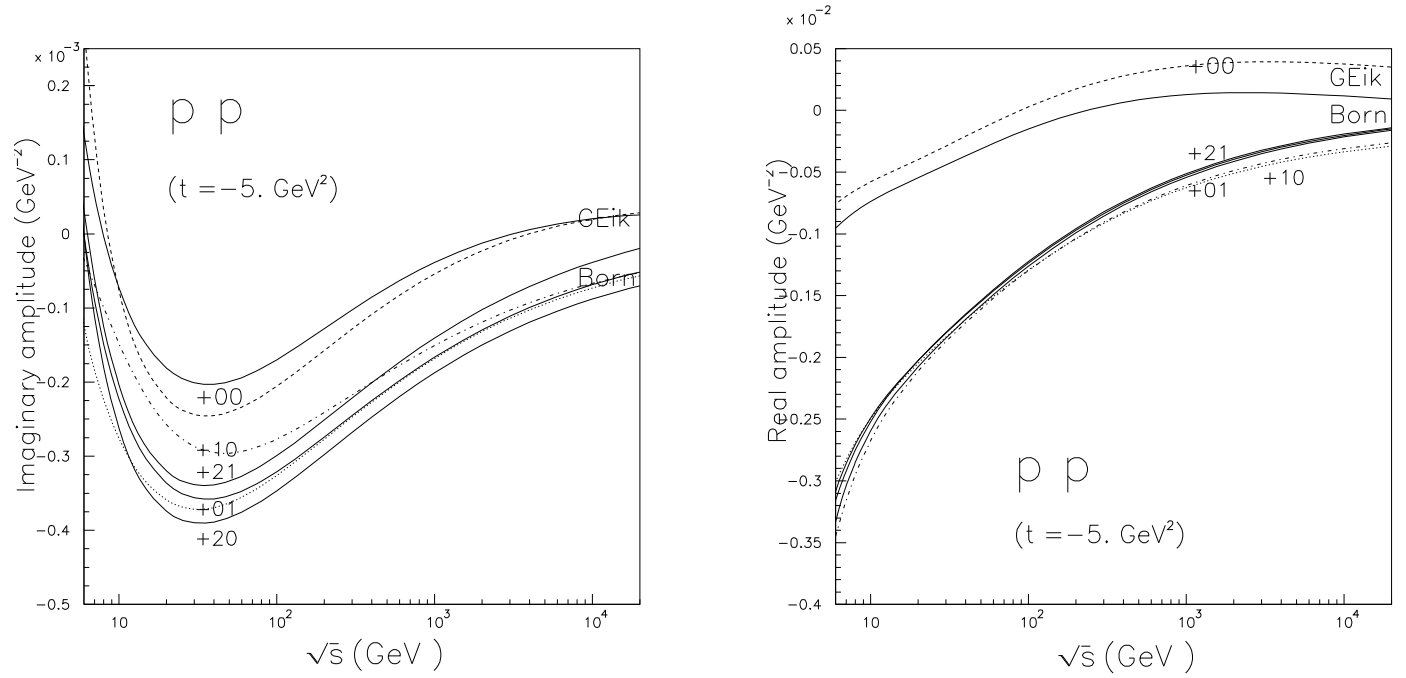

Figure 5: Same as in Fig.1 for $t=-5 . \mathrm{GeV}^{2}$

angular distributions at the ISR, then, taken one by one, the corrections due to the rescattering still increase smoothly with the energy. But, and this is rather surprising, their sum tends towards a constant value, resulting in a GE limit almost parallel (and below) the Born estimation as soon as the energy exceeds $50 \mathrm{GeV}$.

(4) the value $t=-2 \mathrm{GeV}^{2}$ (see Fig. 4) is in a region where strong interferences between the various terms exist; however, one remarks like for $t=-1 \mathrm{GeV}^{2}$, an individual growth of the corrections with the energy and changes of sign resulting in a crossing of the Born and GE limit; otherwise stated, there is a clear global compensation of the corrections at the LHC energy for that transfer.

(5) when $t$ has an intermediate value, beyond the first dip-bump structure $(t=-5$ $\mathrm{GeV}^{2}$, see Fig. 5), in addition to the five values of $\left(n_{+}, n_{-}\right)$necessary to have a good precision when estimating the amplitude, one should also consider the term $(2,1)$. In contrast with the preceding cases, $(i)$ both imaginary and real part of the eikonalized amplitude are above the Born ones (ii) the growth with the energy of the absolute 

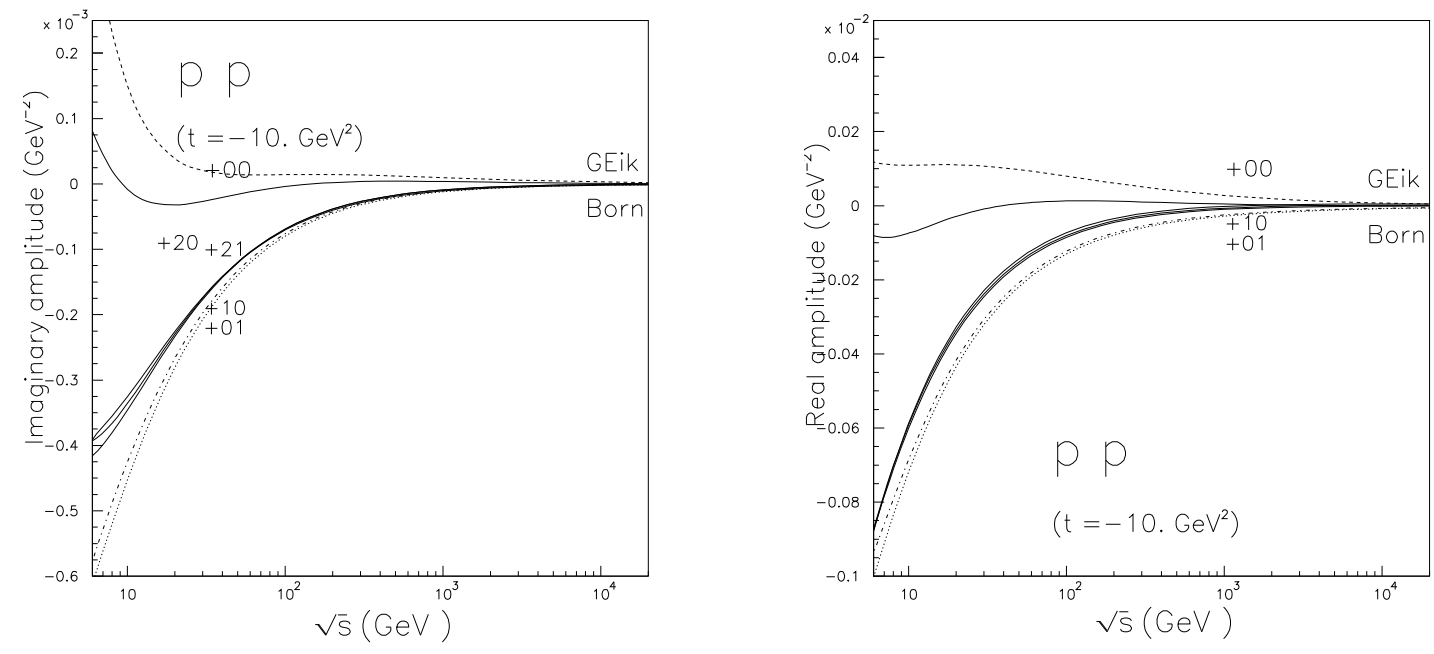

Figure 6: Same as in Fig.1 for $t=-10 . \mathrm{GeV}^{2}$

values of the corrections due to the rescattering which saturates at smaller $|t|$ begins to decrease in the considered energy range (a reminiscence of the dip ?). Finally, we remark that at the highest investigated energy, the eikonalized amplitude (real and imaginary part) is almost zero, but the corrections, though very small, may exceed the Born result by several orders of magnitude. They cannot be neglected since they bring the main contribution to the angular distributions.

(6) when $-t=10 \mathrm{GeV}^{2}$ (see Fig. 6), highest value in the future prospects [7], the various corrections tend asymptotically to become very small at high energy (at the Tevatron and LHC) resulting in a numerical coincidence between the Born and the GE results corresponding to a very small differential cross-section. The same remarks as in the preceding case are valid.

\begin{tabular}{|c|c|c|c|c|}
\hline \multirow[t]{3}{*}{$\left(n_{+} n_{-}\right)$} & & $\begin{array}{c}\text { Born } \\
\left(\mathrm{GeV}^{-2}\right)\end{array}$ & $\begin{array}{c}\text { correction } \\
(\%)\end{array}$ & $\begin{array}{c}\mathrm{GE} \\
\left(\mathrm{GeV}^{-2}\right)\end{array}$ \\
\hline & Imag & 0.752 & & 0.347 \\
\hline & Real & 0.082 & & -0.055 \\
\hline \multirow[t]{2}{*}{$(00)$} & Imag & & -65. & \\
\hline & Real & & -244 & \\
\hline \multirow[t]{2}{*}{ (10) } & Imag & & +14 . & \\
\hline & Real & & +66 . & \\
\hline \multirow[t]{2}{*}{$(20)$} & Imag & & -2 & \\
\hline & Real & & -12 . & \\
\hline \multirow[t]{2}{*}{$(01)$} & Imag & & - & \\
\hline & Real & & +28. & \\
\hline \multirow[t]{2}{*}{ (11) } & Imag & & - & \\
\hline & Real & & 7. & \\
\hline \multirow[t]{2}{*}{$(21),(30)$} & Imag & & - & \\
\hline & Real & & 1. & \\
\hline
\end{tabular}

Table 1. Typical contributions of the main rescattering terms specified by $\left(n_{+} n_{-}\right)$ 
relative to the Born contributions (see the text). An arbitrary criterion of $1 \%$ has been set to limit their number. The energy and transfer are $1000 \mathrm{GeV},-0.5 \mathrm{GeV}^{2}$ respectively. Also quoted are the values of the amplitude computed at the Born level and once the complete generalized eikonalization is performed (GE).

In summary, it appears that the rescattering corrections to the amplitude cannot be neglected especially at high energy and transfer. In addition, the hierarchy of the corrections also depends (as expected) somewhat on the energy and transfer. As a general rule, for the Regge model considered here, one can only be sure that $\left(n_{+}, n_{-}\right)=(0,0)$ brings always the most important contribution and that limiting these indexes by $n_{+}=2$ and $n_{-}=1$ is probably sufficient at not too high energy and transfer.

\section{Discussion and conclusion}

The total cross-section is directly related to the imaginary part of the forward amplitude, hence a part of the conclusions of the preceding section is fully usable. For the $\rho$-ratio, it is less evident to discuss the effects of the rescattering using the preceding considerations on the complex amplitude. The differential cross-section being proportional to the squared modulus of the amplitude, it is not straightforward to visualize the effects on its behavior when adding separately the rescatterings. Its non-linear character obscures the effects we want to investigate. The complete reconstruction of the amplitude requires the knowledge of both the imaginary and real part for all $(s, t)$ inside the experimental ranges (which is not possible from the data) and it is not quite evident that the conclusions of the preceding section on the complex amplitudes still hold for the real observables.

As in [5], when fitting the Dipole or the Monopole Pomeron model with the GE procedure, in the present work, many tests have proven than the $\chi^{2}$ strictly does not change if we overpass $n_{+}=2$ and $n_{+}=1$, keeping more than $3 \times 2=6$ terms in the series $(2)$ (i.e. $\left.\left(n_{-},+n_{-}\right)=(0,0),(1,0),(2,0),(0,1),(1,1),(2,1)\right)$. Further, a poor approximation is realized with two $(0,0)$ and $(1,0)$, or better with three of them $(0,0),(1,0),(2,0)$. That does NOT mean that, if we scrutinize in particular some differential cross-sections at intermediate $|t|$-values and at high energy, one knows which kind of approximation is to be used, because (i) the involved data are scarce and then they are depreciated in the global minimization procedure (ii) the addition of higher terms required in the amplitude may have significant consequences on the angular distribution for some $(s, t)$, meriting a separate study.

The last, but not the least important item we discuss is the use of various approximations.

A mean to see in which kinematical range an approximation is efficient is to examine numerically its consequences on the agreement with fitted observables or, outside the experimentally investigated domain, with the predictions of non-truncated series (GE case), which reproduces the data. We choose the $p p$ angular distributions, 
known at the ISR up to rather large transfers, and extrapolate up to future experimental conditions. We comment our results on the following points.

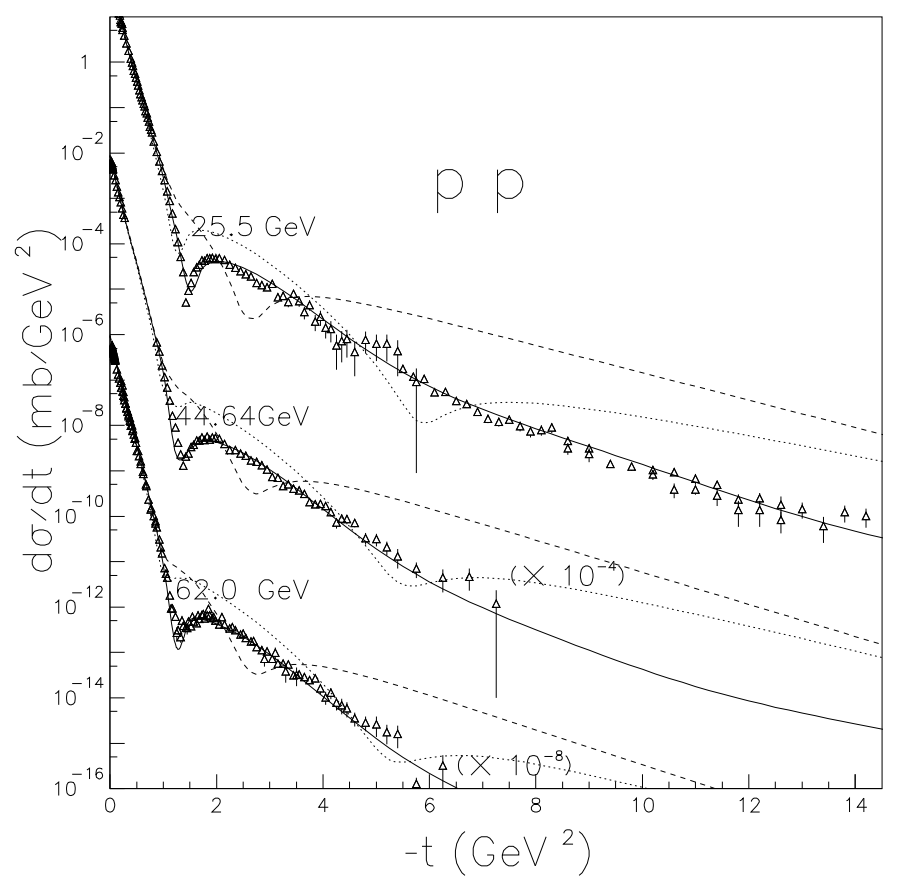

Figure 7: Modifications of a part of the fit in [5] (solid line) when the rescattering series is approximated with the two-Pomeron exchanges exclusively (dashed line) or with the all ten possible two-Reggeon exchanges, i.e. $\left(n_{+}, n_{-}\right)=(0,0)$ (dotted line).

- Assuming only two-Pomeron exchange as a first approximation is a pioneering idea to create the dip [12] and we know in advance it should be insufficient. In our language, one extract the Pomeron-Pomeron contribution from the ten possible two-Reggeon exchanges with $\left(n_{+}, n_{-}\right)=(0,0)$. Fig. 7 is an illustrative example of the inadequacy of this double approximation, for the differential cross-section, as soon as the transfer is not equal zero.

- The next step we consider, is to keep all the ten two-Reggeon exchanges, i.e. when approximating the series $(2)$ with its first term, $\left(n_{+}, n_{-}\right)=(0,0)$. The result is shown in Figs. 7-8. The agreement with the non-truncated series is far from being good. We note a slight improvement with respect to the twoPomeron approximation, especially in reproducing a larger part of the first cone, in creating a dip (although too high, but unfortunately a second dip is also created) and in reducing the differential cross-section at high $|t|$ (which remains still too high).

- We go on further by taking into account in addition all the three-Reggeon exchanges, i.e. limiting the series to the first three terms $\left(n_{+}, n_{-}\right)=(0,0)$ (twoReggeon exchanges) and $\left(n_{+}, n_{-}\right)=(0,1),(1,0)$ (three-Reggeon exchanges). The agreement is better than in the preceding approximation (see Fig. 8) but 


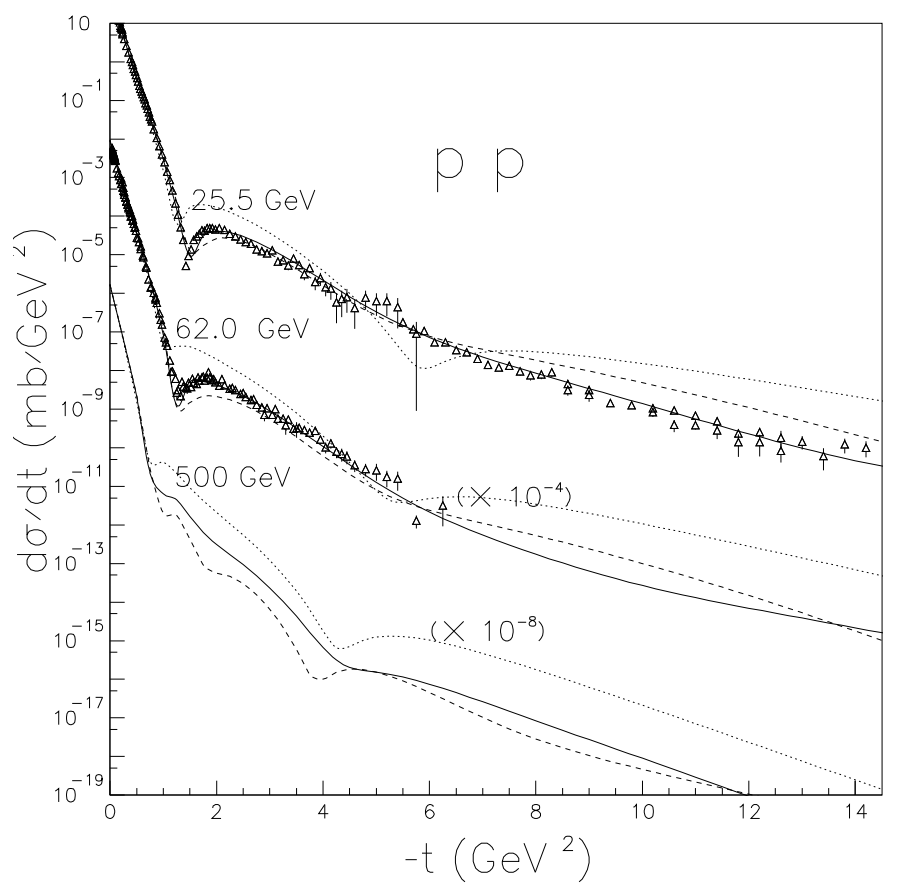

Figure 8: Modifications of a part of the fit in [5] and of prediction at RHIC energy (solid line) when the rescattering series is approximated with the two-Reggeon exchanges alone (dotted line) i.e. $\left(n_{+}, n_{-}\right)=(0,0)$, or when the three-Reggeon exchanges are added, i.e. $\left(n_{+}, n_{-}\right)=(0,0),(10),(01)$ (dashed line).

is still unsatisfying. Here we can only say that all the three-Reggeon exchanges contribute significantly to improve the agreement with the known data and, when these are lacking, to get near the GE results.

- We are in perfect agreement with the statements of the preceding section concerning the number and specificity of exchanges that must be retained to approximate the series with its limit, given here by the generalized eikonalization procedure. From extrapolated differential cross-section calculations, we find that the upper bound of the index $n_{+}$(let us call it $N_{+}$) and, to a lesser extend $N_{-}$, upper bound of $n_{-}$, leading to a convergence towards the GE cross-section, increases with $s$ and mostly with $t$ as indicated in Table 2 , giving rise to a very complex picture of rescatterings in terms of possible diagrams. Whether or not this turns out to be true, only new data can decide of the quality of the extrapolation and its consequences. 


\begin{tabular}{|c|c|c||c|c|}
\hline & $\begin{array}{c}\text { Energy } \\
(\mathrm{GeV})\end{array}$ & $\begin{array}{c}\text { Transfer } \\
\left(\mathrm{GeV}^{2}\right)\end{array}$ & $N_{+}$ & $N_{-}$ \\
\hline \hline RHIC & 200 & 0.0 & 1 & 0 \\
$"$ & $($ or 500) & -0.12 & 1 & 0 \\
$"$ & $"$ & -2.8 & 3 & 1 \\
$"$ & $"$ & -6.0 & 3 & 1 \\
\hline TEVATRON & 2000 & 0.0 & 1 & 0 \\
\hline LHC & 14000 & 0.0 & 2 & 0 \\
$"$ & $"$ & -10. & 5 & 2 \\
\hline
\end{tabular}

Table 2 . Upper indexes of the rescattering series $\left(N_{+}, N_{-}\right)$, necessary to approximate, within a precision of $1 \%$, the GE value of the $p p$ differential cross-section (from the limit of non-truncated series), extrapolated for the energy and transfer of the future experiments [7].

Turning to an other type of approximation, we mention, as a widespread opinion, that one can neglect the $f$ - and the $\omega$ - contributions (and consequently, their rescattering terms) at high energy. We have tested this assertion (giving a sense to the adjective "high"), and found that the $\omega$-Reggeon (with all its rescatterings) is fully negligible only if $\sqrt{s} \gtrsim 500 \mathrm{GeV}$, while for the $f$-Reggeon, the same is true only at an energy higher than a few $\mathrm{TeV}$.

Clearly, limiting the rescatterings to the three- or two-Reggeon exchanges (and a fortiori to the two-Pomeron exchanges) gives a very crude (wrong) estimation of the angular distribution as soon as $-t$ exceeds zero. A correct picture of the rescatterings, compatible with presently available data, requires to limit the series to at least $\left(N_{+}, N_{-}\right)=(2,1)$. Indeed, these six couples of indexes correspond to a quite large number of exchanges (ten for the $(0,0)$ two-Reggeon exchanges, twenty for the $(0,1),(1,0)$ three-Reggeon exchanges, etc... but we remark that the complete list of the all four- and five-Reggeon exchanges is not required).

Finally it is worth pointing out that the substance of the present paper would have been unchanged if we have illustrated it with the GE Monopole Pomeron model instead of the GE Dipole Pomeron model.

Acknowledgements We thank L. Jenkovszky for a critical discussion.

\section{APPENDIX}

Selecting and completing the information given in [5, 9], we collect here the useful formula to understand and perform the rescattering calculations in the "Generalized Eikonalization" (GE) procedure for the "Dipole Pomeron"(DP) model. We emphasize that all the relevant expressions are analytical (they do not require any numerical integration).

\section{A. Input Born in the $s, t$ space. The Dipole Pomeron model}

We focus on the (dimensionless) Born crossing-even and -odd amplitudes $a_{ \pm}(s, t)$ of 
the $p p$ and $\bar{p} p$ reactions

$$
a_{p p ; B o r n}^{\bar{p} p}(s, t)=a_{+}(s, t) \pm a_{-}(s, t)
$$

starting point to get the eikonalized amplitudes $A(s, t)=A_{p p}^{\bar{p} p}(s, t)$ used to fit : i) the total cross-sections

$$
\sigma_{\mathrm{tot}}=\frac{4 \pi}{s} \Im \mathrm{m} A(s, t=0)
$$

ii) the differential cross-sections

$$
\frac{d \sigma}{d t}=\frac{\pi}{s^{2}}|A(s, t)|^{2}
$$

iii) and the ratios of the real to the imaginary forward amplitudes

$$
\rho=\frac{\Re \mathrm{e} A(s, t=0)}{\Im \mathrm{m} A(s, t=0)} .
$$

The crossing even part in the Born amplitude is a Pomeron, to which a $f$-Reggeon is added, while the crossing odd part is an Odderon (plus an $\omega$-Reggeon)

$$
a_{+}(s, t)=a_{P}(s, t)+a_{f}(s, t), \quad a_{-}(s, t)=a_{O}(s, t)+a_{\omega}(s, t) .
$$

For simplicity, in our DP model, the two Reggeons have been taken in the standard form

$$
a_{R}(s, t)=a_{R} \eta_{R} \tilde{s}^{\alpha_{R}(t)} e^{b_{R} t}, \quad(R=f \text { and } \omega), \quad \eta_{f}=1, \eta_{\omega}=i,
$$

with linear trajectories

$$
\alpha_{R}(t)=\alpha_{R}(0)+\alpha_{R}^{\prime} t, \quad(R=f \text { and } \omega) .
$$

Here a "dipole" is chosen for the Pomeron (i.e. a linear combination of a simple pole with a double pole)

$$
a_{P}(s, t)=a_{P}^{(D)}(s, t)=a_{P} \tilde{s}^{\alpha_{P}(t)}\left[e^{b_{P}\left(\alpha_{P}(t)-1\right)}\left(b_{P}+\ell n \tilde{s}\right)+d_{P} \ell n \tilde{s}\right] .
$$

The Odderon is obtained with the same requirements as for the Pomeron, but multiplied by a convenient damping factor killing it at $t=0$ in order to respect the common knowledge

$$
a_{O}(s, t)=(1-\exp \gamma t) i a_{O}^{(D)}(s, t)
$$

i.e. the amplitude on the r.h.s. $a_{O}^{(D)}(s, t)$ is constructed along the same lines as $a_{P}^{(D)}(s, t)$, changing only the parameters. As usual,

$$
\tilde{s}=\frac{s}{s_{0}} e^{-i \frac{\pi}{2}}, \quad\left(s_{0}=1 \mathrm{GeV}^{2}\right)
$$

\footnotetext{
${ }^{8}$ Once again, $+(-)$ correspond to $\bar{p} p(p p)$ process; note that the normalization of [5] is used and that the coupling constants $a_{P}, a_{O}, a_{f}, a_{\omega}$ are reals.
} 
enforces $s-u$ crossing and $\alpha_{i}(t)$ are the trajectories taken, for simplicity, of the linear form

$$
\alpha_{i}(t)=1+\delta_{i}+\alpha_{i}^{\prime} t,(i=P, O)
$$

and verifying the unitarity constraints

$$
\delta_{P} \geq \delta_{O}, \quad \text { and } \quad \alpha_{P}^{\prime} \geq \alpha_{O}^{\prime} .
$$

\section{B. Born amplitude in the $s, b$ space}

In eikonal models, the scattering amplitudes are expressed in the impact-parameter (" $b ")$ representation ( $s, b$ space). First, one defines the Fourier-Bessel's (F-B) transform of the Born amplitude

$$
h_{p p}^{\bar{p} p}(s, b)=\frac{1}{2 s} \int_{0}^{\infty} a_{p p ; B o r n}^{\bar{p} p}\left(s,-q^{2}\right) J_{0}(b q) q d q \quad \text { with } \quad q=\sqrt{-t} .
$$

This is related to the eikonal function (" eikonal" for brevity) by

$$
\chi_{p p}^{\bar{p} p}(s, b)=2 h_{p p}^{\bar{p} p}(s, b) .
$$

In all eikonalization procedures, one first derives the eikonalized amplitude in the b-representation $H_{p p}^{\bar{p} p}(s, b)$; the inverse F-B transform leads then to the usual eikonalized amplitude in the $s, t$ space

$$
A_{p p}^{\bar{p} p}(s, t)=2 s \int_{0}^{\infty} H_{p p}^{\bar{p} p}(s, b) J_{0}(b \sqrt{-t}) b d b .
$$

The main technical problem of eikonalization is the derivation of $H_{p p}^{\bar{p} p}(s, b)$ once $h_{p p}^{\bar{p} p}(s, b)$ are given (for details, see [9]).

Although not required in practice since it is integrated when eikonalizing, and consequently it is an intermediate quantity, we give here the expression of the Born amplitude in the impact parameter representation (half of the eikonal function)

$$
h_{p p}^{\bar{p} p}(s, b)=\frac{1}{2} \chi_{p p}^{\bar{p} p}(s, b)=h_{f}(s, b)+h_{P}(s, b) \pm\left[h_{O}(s, b)+h_{\omega}(s, b)\right],
$$

with the DP model, defined above.

For the secondary Reggeons, we obtain

$$
h_{R}(s, b)=\frac{1}{2} \eta_{R} a_{R} \frac{\tilde{s}^{\alpha_{R}(0)}}{s} \frac{\exp \left(\frac{-b^{2}}{4 B_{R}}\right)}{2 B_{R}}=\frac{1}{2} \chi_{R}(s, b),
$$

with (see also $(A 6-7))$

$$
B_{R} \equiv B_{R}(s)=\alpha_{R}^{\prime} \ln \tilde{s}+b_{R}, \quad R=(f, \omega), \quad \eta_{f}=1, \eta_{\omega}=i .
$$

The Pomeron dipole splits into 2 components

$$
\begin{aligned}
h_{P}(s, b) & =\frac{-i a_{P}}{4 \alpha_{P}^{\prime} s_{0}}\left(e^{r_{1, P} \delta_{P}-\frac{b^{2}}{4 B_{1, P}}}+d_{P} e^{r_{2, P} \delta_{P}-\frac{b^{2}}{4 B_{2, P}}}\right) \\
& \equiv \frac{1}{2} \chi_{P}(s, b)=\frac{1}{2}\left(\chi_{P 1}(s, b)+\chi_{P 2}(s, b)\right) .
\end{aligned}
$$


The Odderon dipole with its damping factor yields

$$
\begin{aligned}
h_{O}(s, b) & =\frac{a_{O}}{4 \alpha_{O}^{\prime} s_{0}}\left(e^{r_{1, O} \delta_{O}-\frac{b^{2}}{4 B_{1, O}}}+d_{O} e^{r_{2, O} \delta_{O}-\frac{b^{2}}{4 B_{2, O}}}\right) \\
& -\frac{a_{O}}{4 \alpha_{O}^{\prime} s_{0}}\left(e^{r_{1, O} \delta_{O}-\frac{b^{2}}{4 B_{1, O}}} \frac{B_{1, O}}{\widehat{B_{1, O}}}+d_{O} e^{r_{2, O} \delta_{O}-\frac{b^{2}}{4 B_{2, O}}} \frac{B_{2, O}}{\widehat{B_{2, O}}}\right) \\
& \equiv \frac{1}{2} \chi_{O}(s, b)=\frac{1}{2}\left(\chi_{O 1}(s, b)+\chi_{O 2}(s, b)+\widetilde{\chi_{O 1}}(s, b)+\widetilde{\chi_{O}}(s, b)\right) .
\end{aligned}
$$

We have defined (see also $(A 8-11))$

$$
r_{1, J} \equiv r_{1, J}(s)=\ell n \tilde{s}+b_{J}, r_{2, J} \equiv r_{2, J}(s)=\ell n \tilde{s},(J=P, O),
$$

and

$$
\begin{aligned}
B_{i, P} & \equiv B_{i, P}(s)=\alpha_{P}^{\prime} r_{i, P}, \quad B_{i, O} \equiv B_{i, O}(s)=\alpha_{O}^{\prime} r_{i, O} \\
\widetilde{B_{i, O}} & \equiv \widetilde{B_{i, O}}(s)=\alpha_{O}^{\prime} r_{i, O}+\gamma,(i=1,2) .
\end{aligned}
$$

\section{Rescattering Series}

We rewrite the rescattering series (2) (part of the eikonalized amplitude added to the Born contribution (1))

$$
A_{p p, r e s c a t}^{\bar{p} p}(s, t)=\sum_{n_{+}=0}^{\infty} \sum_{n_{-}=0}^{\infty} a_{p p ; n_{+}, n_{-}}^{\bar{p} p}(s, t)
$$

with the $\left(n_{+}, n_{-}\right)$term in the general case of three parameters $\left(\lambda_{ \pm}, \lambda_{0}\right)$ of the GE procedure

$$
\begin{aligned}
a_{p p ; n_{+}, n_{-}}^{\bar{p} p}(s, t) & =i s \frac{\left(i \lambda_{+}\right)^{n_{+}}\left( \pm i \lambda_{-}\right)^{n_{-}}}{\left(n_{+}+n_{-}+2\right) !} \\
& \times\left(F_{n_{+}, n_{-}}(z) \cdot I+F_{n_{-}, n_{+}}(z) \cdot I I+G_{n_{+}, n_{-}}(z) \cdot I I I\right),
\end{aligned}
$$

where the hypergeometric function ${ }_{2} F_{1}$ has been introduced in $F$ and $G$, functions of the argument $z=\frac{\lambda_{0}^{2}}{\lambda_{+} \lambda_{-}}$

$$
\begin{aligned}
& F_{n_{ \pm}, n_{\mp}}(z)=z\left(n_{ \pm}+1\right) \cdot{ }_{2} F_{1}\left(1-n_{\mp},-n_{ \pm} ; 2 ; z\right) \cdot\left(1-\delta_{n_{\mp}, 0}\right)+\delta_{n_{\mp}, 0}, \\
& G_{n_{+}, n_{-}}(z)={ }_{2} F_{1}\left(-n_{-},-n_{+} ; 1 ; z\right) .
\end{aligned}
$$

The inverse Fourier-Bessel transforms are the following three functions in the $s, t$ space :

$$
\begin{gathered}
I=\lambda_{+} \sum_{\ell=0}^{n_{+}+2} \sum_{m=0}^{n_{-}}\left(\begin{array}{c}
n_{+}+2 \\
\ell
\end{array}\right)\left(\begin{array}{c}
n_{-} \\
m
\end{array}\right) \cdot \operatorname{Int}_{n_{+}+2-\ell, n_{-}-m, \ell, m}(s, t), \\
I I=\lambda_{-} \sum_{\ell=0}^{n_{+}} \sum_{m=0}^{n_{-}+2}\left(\begin{array}{c}
n_{+} \\
\ell
\end{array}\right)\left(\begin{array}{c}
n_{-}+2 \\
m
\end{array}\right) \cdot \operatorname{Int}_{n_{+}-\ell, n_{-}+2-m, \ell, m}(s, t), \\
I I I= \pm 2 \frac{\lambda_{+} \lambda_{-}}{\lambda_{0}} \sum_{\ell=0}^{n_{+}+1} \sum_{m=0}^{n_{-}+1}\left(\begin{array}{c}
n_{+}+1 \\
\ell
\end{array}\right)\left(\begin{array}{c}
n_{-}+1 \\
m
\end{array}\right) \cdot \operatorname{Int}_{n_{+}+1-\ell, n_{-}+1-m, \ell, m}(s, t) .
\end{gathered}
$$


$\left(\begin{array}{l}n \\ k\end{array}\right)$ is the binomial coefficient and $\operatorname{Int}(s, t)$ is the integral over the four eikonals defined above i.e.

$$
\text { Int }{ }_{\lambda, \mu, \ell, m}(s, t)=\int_{0}^{\infty} \chi_{P}^{\lambda}(s, b) \chi_{O}^{\mu}(s, b) \chi_{f}^{\ell}(s, b) \chi_{\omega}^{m}(s, b) J_{0}(b \sqrt{-t}) b d b .
$$

When the dipole Odderon does contain a damping factor at $t=0$, this integral writes

$$
\begin{aligned}
& \operatorname{Int}_{\lambda, \mu, \ell, m}(s, t)=C \sum_{\lambda^{\prime}=0}^{\lambda} \sum_{\sigma=0}^{\mu} \sum_{\mu^{\prime}=0}^{\mu-\sigma} \sum_{\nu=0}^{\sigma}\left(\begin{array}{c}
\lambda \\
\lambda^{\prime}
\end{array}\right)\left(\begin{array}{c}
\mu \\
\sigma
\end{array}\right)\left(\begin{array}{c}
\mu-\sigma \\
\mu^{\prime}
\end{array}\right)\left(\begin{array}{c}
\sigma \\
\nu
\end{array}\right) \\
& \times \exp \left[r_{1, P} \delta_{P}\left(\lambda-\lambda^{\prime}\right)+r_{2, P} \delta_{P} \lambda^{\prime}+r_{1, O} \delta_{O}\left(\mu-\mu^{\prime}-\nu\right)+r_{2, O} \delta_{O}\left(\mu^{\prime}+\nu\right)\right] \\
& \times d_{P}^{\lambda^{\prime}} d_{O}^{\mu^{\prime}+\nu}\left(-\frac{B_{1, O}}{\widetilde{B_{1, O}}}\right)^{\sigma-\nu}\left(-\frac{B_{2, O}}{\widetilde{B_{2, O}}}\right)^{\nu} \cdot \operatorname{int}(s, t),
\end{aligned}
$$

where

$$
\begin{gathered}
C \equiv C(s)=\left(\frac{-i a_{P}}{2 \alpha_{P}^{\prime} s_{0}}\right)^{\lambda}\left(\frac{a_{O}}{2 \alpha_{O}^{\prime} s_{0}}\right)^{\mu}\left(\frac{a_{f} \tilde{s}^{\alpha_{f}(0)}}{2 B_{f} s}\right)^{\ell}\left(\frac{i a_{\omega} \tilde{s}^{\alpha_{\omega}(0)}}{2 B_{\omega} s}\right)^{m} ; \\
\operatorname{int}(s, t)=\int_{0}^{\infty} \exp \left(\frac{-D b^{2}}{4}\right) J_{0}(b \sqrt{-t}) b d b=\frac{2}{D} \exp \left(\frac{t}{D}\right) \\
D \equiv D(s)=\frac{\lambda-\lambda^{\prime}}{B_{1, P}}+\frac{\lambda^{\prime}}{B_{2, P}}+\frac{\mu-\sigma-\mu^{\prime}}{B_{1, O}}+\frac{\mu^{\prime}}{B_{2, O}}+\frac{\sigma-\nu}{\widetilde{B_{1, O}}}+\frac{\nu}{\widetilde{B_{2, O}}}+\frac{\ell}{B_{f}}+\frac{m}{B_{\omega}} .
\end{gathered}
$$

\section{References}

[1] V.N. Gribov, Sov. Phys. JETP 26, 414 (1968)

[2] K.A. Ter-Martirosyan, Sov. Nucl. Phys. 10, 1047 (1969)

[3] T.T. Chou, C.N. Yang, Phys. Rev. 170, 1591 (1968);

A.A. Migdal, A.M. Poliakov, K.A. Ter-Martirosyan, Phys. Lett. 48 B, 239 (1974);

J.L. Cardy, Nucl. Phys. B 75, 413 (1974);

M.D.J. Abarbanel, J.B. Bronzan, R.L. Sugar, A.R. White, Phys. Rep. 21 C, 120 (1975);

C. Bourrely, J.S. Soffer, T.T. Wu, Phys. Rev. D 19, 3249 (1979);

S.M. Troshin, N.E. Tyurin, Sov. Jour. Part. Nucl. 15, 25 (1984);

L.L. Jenkovszky, B.V. Struminsky, A.N. Wall, Sov. Jour. Part. Nucl. 19, 180 (1988) 
[4] T.T. Chou, C.N. Yang, Phys. Rev. Lett. 20, 1213 (1968);

T.T. Chou, C.N. Yang, Phys. Rev. D 17, 1889 (1978);

P.E. Volkovitskij, A.M. Lapidus, V.I. Lisin, K.A. Ter-Martirosyan, Sov. J. Nucl. Phys. 24, 648 (1976);

C. Bourrely, J.S. Soffer, T.T. Wu, Nucl. Phys. B 247, 15 (1984);

[5] P. Desgrolard, M. Giffon, E. Martynov, E. Predazzi, Eur. Phys. J. C 16, 499 $(2000)$

[6] P. Desgrolard, L. Jenkovszky, Ukr. J. Phys. 41-4, 396 (1996);

P. Desgrolard, Ukr. J. Phys. 42-3, 2661 (1997)

[7] S.B. Nurushev, in Proceedings of the International Conference on Elastic and Diffractive Scattering (VIII ${ }^{\text {th }}$ EDS Blois Workshop), Protvino, Russia, 1999, eds. V.A. Petrov and A.V. Prokudin (World Scientific Publishing, 2000) p.313; TOTEM, CERN/LHCC 97-49, 1997 and 99-7, 1999;

S. Weisz, talk at International Conference on Elastic and Diffractive Scattering (VIII $^{\text {th }}$ EDS Blois Workshop), Protvino, Russia (June 28-July 2, 1999);

A.D. Martin, Summary talk at the first Worshop on Forward Physics and Luminosity Determination at the LHC, Helsinki, (October 31-November 3, 2000); arXiv: hep-ph/0103296

[8] M.Froissart, Phys. Rev. 123, 1053 (1961);

A. Martin, Phys. Rev. 129, 432 (1963), Nuovo Cim. 29, 993 (1963)

[9] P. Desgrolard, M. Giffon, E. Martynov, E. Predazzi, Eur. Phys. J. C 14, 683 $(2000)$

[10] L.L. Jenkovszky, F. Phys. 34, 702 (1986)

[11] V.A. Petrov, A.V. Prokudin, in Proceedings of the International Conference on Elastic and Diffractive Scattering (VII ${ }^{\text {th }}$ Blois Workshop), Protvino, Russia, 1999, eds. V.A. Petrov and A.V. Prokudin (World Publishing, 2000) p.95; arXiv: hep-ph/9912245

[12] See for example : P.D.B. Collins, F. Gault, Phys. Lett. 47 B, 171 (1973) 